Full length article

\title{
Cladding waveguide splitters fabricated by femtosecond laser inscription in Ti:Sapphire crystal
}

\author{
Yingying Ren ${ }^{\mathrm{a}, \mathrm{b}, *}$, Limu Zhang ${ }^{\mathrm{a}}$, Hongguang Xing a ${ }^{\mathrm{a}}$, Carolina Romero ${ }^{\mathrm{d}}$, Javier R. Vázquez de Aldana ${ }^{\mathrm{d}}$, \\ Feng Chen ${ }^{\mathrm{c}, *}$ \\ a Shandong Provincial Key Laboratory of Optics and Photonic Device, School of Physics and Electronics, Shandong Normal University, Jinan 250014, China \\ ${ }^{\mathrm{b}}$ Institute of Data Science and Technology, Shandong Normal University, Jinan 250014, China \\ ${ }^{\text {c } S c h o o l ~ o f ~ P h y s i c s, ~ S t a t e ~ K e y ~ L a b o r a t o r y ~ o f ~ C r y s t a l ~ M a t e r i a l s, ~ S h a n d o n g ~ U n i v e r s i t y, ~ J i n a n ~ 250100, ~ C h i n a ~}$ \\ ${ }^{\mathrm{d}}$ Laser Microprocessing Group, Departamento de Física Aplicada, Universidad de Salamanca, Salamanca 37008, Spain
}

\section{A R T I C L E I N F O}

\section{Article history:}

Received 7 September 2017

Received in revised form 22 November 2017

Accepted 4 January 2018

\section{Keywords:}

Optical cladding waveguides

Beam splitters

Femtosecond laser inscription

Ti:Sapphire crystal

\begin{abstract}
A B S T R A C T
Highly-compact devices capable of beam splitting are intriguing for a broad range of photonic applications. In this work, we report on the fabrication of optical waveguide splitters with rectangular cladding geometry in a Ti:Sapphire crystal by femtosecond laser inscription. Y-splitters are fabricated with $30 \mu \mathrm{m}$ $\times 15 \mu \mathrm{m}$ and $50 \mu \mathrm{m} \times 25 \mu \mathrm{m}$ input ends, corresponding to two $15 \mu \mathrm{m} \times 15 \mu \mathrm{m}$ and $25 \mu \mathrm{m} \times 25 \mu \mathrm{m}$ output ends, respectively. The full branching angle $\theta$ between the two output arms are changing from $0.5^{\circ}$ to $2^{\circ}$. The performances of the splitters are characterized at $632.8 \mathrm{~nm}$ and $1064 \mathrm{~nm}$, showing very good properties including symmetrical output ends, single-mode guidance, equalized splitting ratios, all-angle-polarization light transmission and intact luminescence features in the waveguide cores. The realization of these waveguide splitters with good performances demonstrates the potential of such promising devices in complex monolithic photonic circuits and active optical devices such as miniature tunable lasers.
\end{abstract}

(c) 2018 Elsevier Ltd. All rights reserved.

\section{Introduction}

Optical waveguides are devices that confine the light propagating effectively within a micro- or submicrometric volume by total internal reflection [1]. Benefiting from their compactness, waveguide structures play critical roles as the basic elements in photonic networks, offering unique platforms for the realization of multiple functions in on-chip circuits. The past decade saw the rapid development of optical waveguides. On the one hand, two- or threedimensional passive waveguiding components (e.g. optical switches, couplers and splitters) with compact architectures, excellent performance and on-demand beam tailoring abilities can be rapidly established by modern micromachining technologies [2-7]. On the other hand, in connection to the progress in achieving versatile high-quality optical materials, the rapid development of optimized waveguides preserving high-fidelity optical features of the substrates leads to the emergence of integrated circuits with a broad spectrum of active applications

* Corresponding authors at: Shandong Provincial Key Laboratory of Optics and Photonic Device, School of Physics and Electronics, Shandong Normal University, Jinan 250014, China (Y. Ren).

E-mail addresses: ryywly@sdnu.edu.cn (Y. Ren), drfchen@sdu.edu.cn (F. Chen). covering amplifiers, frequency converters, lasers and so on [811]. Moreover, with respect to the bulk, some features of the substrate wafer could be enhanced to a certain extent in the waveguides owing to their high compactness, enabling the realization of highly efficient miniature platforms [12].

Among the aforementioned guiding devices, waveguide splitters based on Y-branch structures, featuring with the capability of reformatting the single input into several output beams, are important elements in constructing photonic integrated circuits for a variety of applications including, but not limited to, power splitters, interferometers, multiplexers, hybrid-integrated microfluidic devices and laser generation systems [13-17]. Much effort has been devoted to the development of such waveguide splitters. Ever since the pioneering reports in the mid 90's [18], femtosecond laser inscription (FLI) has emerged as an unprecedented micro-structuring technique to fabricate optical waveguide structures in numerous transparent materials. Due to the extremely high peak intensity that is achieved in ultrashort laser pulses, FLI offers distinct advantages in many aspects including high precision, repeatability, variety of materials that can be processed and, more importantly, the ability for mask-less 3D processing of complex photonic networks [19-23]. During the procedure of FLI, one of the most intriguing consequences of the nonlinear energy 
transfer occurring at the laser focus is the controlled and localized modification of the refractive index (RI) of the material, which enables the formation of waveguide structures in the substrates. It has been proved that the femtosecond ( $\mathrm{fs}$ ) laser induced RI alteration in the focal volume could be either positive or negative. Based on the mechanism of positive RI change, so called Type I waveguide structures can be written directly in the materials with single- or multi-scan techniques, providing a convenient way to implement 3D waveguide splitters. This method has been proved to be suitable to glass [24] and polymers [25], but restricted to only a few crystalline substrates such as $\mathrm{LiNbO}_{3}$ [26] and BGO [27]. For most of crystalline media, negative RI modifications are typically induced in the laser damage tracks. Thus, double-line waveguide that exploits RI increase due to stress fields in the vicinity of low-index tracks can be fabricated (Type II). Waveguide beam splitters have been successfully fabricated with this approach [28]. Unfortunately, in spite of its advantages, such as the variety of materials in which they can be implemented and the simplicity of the manufacturing, its applicability in photonic circuits is often blocked by the polarization-selective guiding behavior. Moreover, for a better overlap of the stress-induced high index regions of two tracks, the waveguide volume is limited to tens of micrometers (typically $10-20 \mu \mathrm{m}$ ), which could neither enable the guidance of long wavelengths (e.g., mid-IR) nor ensure a high coupling efficiency with the different dimensions of light beams from various sources (e.g., the commercial fibers). A possible solution to these limitations is to employ the model of depressed-cladding waveguide structures (Type III). With an architecture that consists of a quasi-continuous barrier (low-index damage tracks) and an intact core region, depressed cladding waveguides show excellent features such as arbitrary geometry and dimensions, well-preserved guiding core, strong light confinement, low propagation loss and, more attractively, two-dimensional (2D) guidance without strong polarization dependence, making this approach advantageous for construction of functional photonic devices. Based on the cladding geometry, superficial splitters with rectangular cross-sections has been fabricated in Nd:YAG [17] and $\mathrm{LiTaO}_{3}$ crystals [29]. These structures possess good properties for both passive and active applications.

Ti:Sapphire crystal is one of the most attractive laser media with large gain bandwidth. Waveguide splitters built in Ti:Sapphire crystals could serve as passive elements in integrated optical circuits. More attractively, combined with the excellent optical features of Ti:Sapphire, the cladding splitters is promising as miniature devices for applications in active regimes as wide spectral luminescence and broadband tunable laser systems with multiple signals generating simultaneously.

In this work, we demonstrate, for the first time, cladding Ybranch waveguides in Ti:Sapphire fabricated by using FLI for beam splitting. The guiding and splitting performances of these cladding structures are investigated experimentally and numerically in the visible and near-infrared for two orthogonal polarizations (TE and $\mathrm{TM})$.

\section{Experimental details}

\subsection{Splitter fabrication by FLI}

Fig. 1(a) depicts schematically the system for the fabrication of waveguide splitters. An amplified Ti:Sapphire laser system (Spitfire, Spectra Physics, USA) is used for waveguide fabrication, delivering $1 \mathrm{kHz}, 120 \mathrm{fs}$ pulses with a central wavelength of $795 \mathrm{~nm}$. By utilizing a set of half-wave plate and a polarizer followed by a calibrated neutral density filter, the laser beam is adjusted to be lin- early polarized and pulse energy is reduced to $1.2 \mu \mathrm{J}$. An approximately $0.15 \mathrm{wt}$. $\% \mathrm{Ti}_{2} \mathrm{O}_{3}$ doped Ti:Sapphire crystal is applied as substrate. After being cut into a dimension of $10 \mathrm{~mm}(\mathrm{a}) \times 2 \mathrm{~mm}$ (b) $\times 10 \mathrm{~mm}$ (c) and polished to optical quality, the sample is mounted onto a motorized 3D translation stage. The inscribing fs-laser is focused into the sample through its top surface (10 $\mathrm{mm}$ (a) $\times 10 \mathrm{~mm}$ (c)) using a $20 \times$ microscope objective (N.A. $=0.4)$. By controlling the 3D stage with a computer, the sample is translated through the laser focus perpendicularly to the beam propagation axis. A translation speed of $500 \mu \mathrm{m} / \mathrm{s}$ is selected which is large enough to avoid the formation of cracks in the sample and small enough to ensure an appropriate RI modification within the focal volume. Under these conditions, the damage track has an axial length of 10 microns (along b-axis) and a lateral diameter of 1.5 microns (along c-axis). Additionally, in order to construct quasi-continuous cladding boundaries, the lateral separation between adjacent scans is set to $3 \mu \mathrm{m}$.

$3 \mathrm{D}$ schematic diagram of the 1 -to- 2 rectangular cladding splitter is shown in Fig. 1(b), where red tracks represent the fs-laser induced tracks with negative RI alteration. Fig. 1(c)-(d) illustrate the front view (corresponding to the input end), top view and back view (output end) of the splitter. The input facets of the splitters in this work are designed to be rectangular (with a width two times the height of the waveguide) and thus two square outputs are achieved. Such a rectangular splitter is easier to realize than the circular one by using FLI. During fabrication process, the paths of the tracks are controlled such that a $3-\mathrm{mm}$ straight bus (i.e. the input arm) along the a-axis is followed by two divergent identical splitting sections forming the fan-out arms. The cladding structures are fabricated in the sample with their lower boundaries located around $260 \mu \mathrm{m}$ beneath the sample surface. In this work, four 1-to-2 beam splitters are fabricated with different sizes, which are $30 \mu \mathrm{m} \times 15 \mu \mathrm{m}$ for the input end of the first splitter (hereafter referred to Sp1) and $50 \mu \mathrm{m} \times 25 \mu \mathrm{m}$ for the others (Sp2-Sp4), corresponding to $15 \mu \mathrm{m} \times 15 \mu \mathrm{m}$ and $25 \mu \mathrm{m} \times 25 \mu \mathrm{m}$ output ends, respectively. The central separation of the two output ends depends on the full branching angle $\theta$ between the two arms, which is arranged as $0.5^{\circ}$ for $\mathrm{Sp} 1$ and $0.5^{\circ}, 1^{\circ}, 2^{\circ}$ for Sp2-Sp4, respectively. As a consequence, separations between $75 \mu \mathrm{m}$ and $269 \mu \mathrm{m}$ are achieved at the end face.

\subsection{Splitting performance characterization}

After irradiation, the resulting splitters are investigated with a metalloscope (Axio Imager, Carl Zeiss) operating in transmission mode to preliminarily assess the fs-laser induced modifications. For further characterizing the splitting performances of the splitters, visible $(632.8 \mathrm{~nm})$ and near-infrared $(1064 \mathrm{~nm})$ lasers are focused and coupled into the input arms with a typical end-face coupling arrangement. Both the modal distributions at transverse electric and transverse magnetic polarizations are measured. The 1064-nm light propagation of the single-mode splitter is simulated by the commercial program BeamPROP (Rsoft $\odot$, Inc) based on the finite difference beam propagation method. To investigate the power splitting ratio, which is an essential index for beam splitters, the output power from each exiting port is measured separately and then compared. Moreover, we also measured the total output power of each structure at arbitrary linear polarization in order to investigate the polarization-dependence of the splitters. The total attenuations of the waveguide splitters including propagation losses and splitting losses at $1064 \mathrm{~nm}$ are also determined based on the end-face coupling method. By directly detecting the incident and output beam powers, the total losses of the splitters can be expressed as follows: 

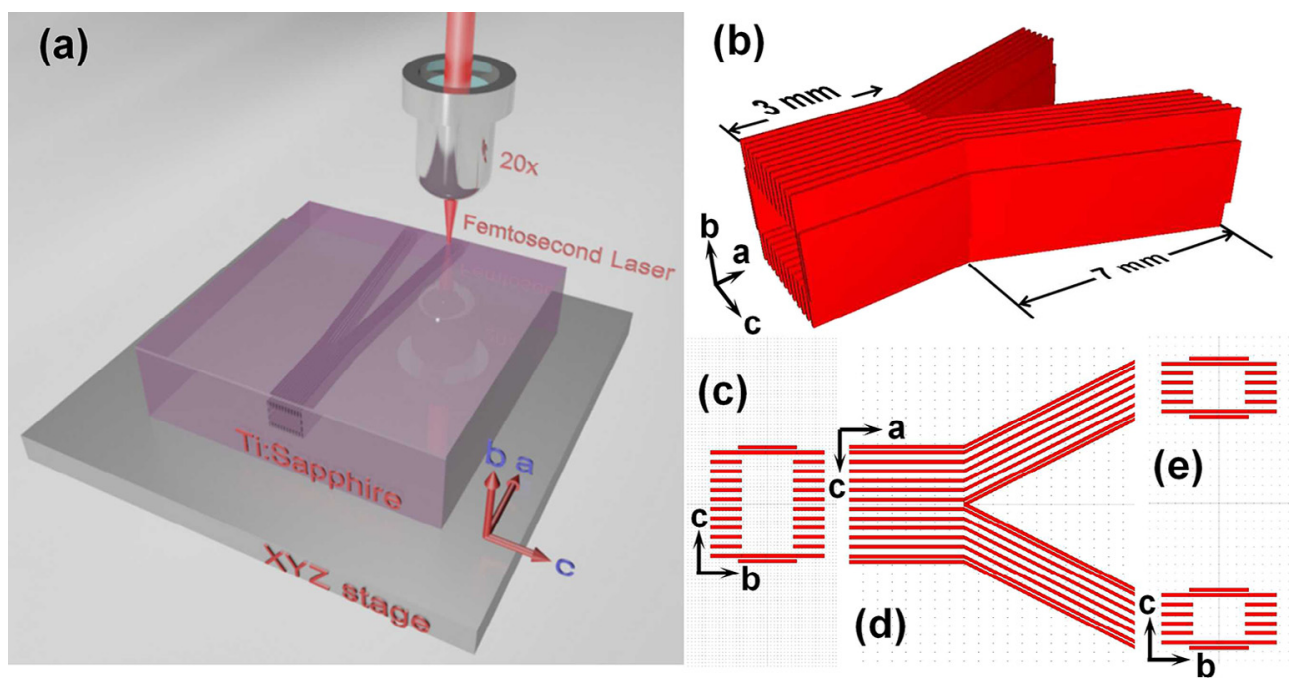

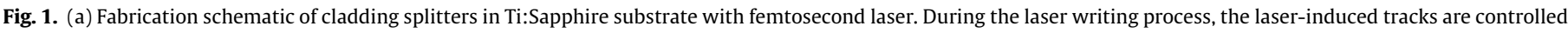

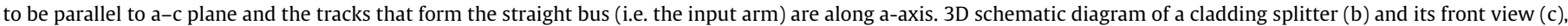
top view (d) as well as back view (e). (c) and (e) are corresponding to the input and output end of the splitter. A, b and c-axis are crystal axes.

$\alpha=-10 \log _{10}\left(\frac{P_{\text {out }}}{\eta P_{\text {in }} T_{o b j}^{2}(1-R)^{2}}\right)$

in which $P_{\text {in }}$ and $P_{\text {out }}$ correspond to the input and output laser powers, respectively. $\eta$ is coupling efficiency between pump beam mode and waveguide mode; $T_{o b j}$ is the transmittance of the microscope objective lens; $R$ is the Fresnel reflection coefficient at each waveguide-air interface.

\subsection{Confocal micro-luminescence measurements}

The room-temperature confocal micro-photoluminescence ( $\mu$-PL) properties of the fabricated structures in Ti:Sapphire are investigated using a confocal microscope (Nanofinder FLEX2. Tokyo Instruments, Inc.). With Sp1 as a representative, the 500 $\mathrm{nm}$ excitation laser light generated from frequency doubling of a Ti:Sapphire laser (Maitai HP, Spectra-Physics) is focused onto the cross sections of the structure and the $\mathrm{Ti}^{3+}$ fluorescence emission signals are collected by applying a spectrometer (DU420A-OE, ANDOR). 2D images and 1D profile of the integrated $\mu$-PL spectral intensity are obtained along with the $\mu$-PL spectral distribution in the unmodified bulk region, the laser-induced tracks and the waveguide area.

\section{Results and discussion}

Fig. 2 presents the microscopic image of input facet and output facet of each splitter (Sp1-Sp4) observed with transmitted illumination light. As can be clearly seen, well-defined rectangular claddings composed of distinct laser induced tracks are established in the Ti:Sapphire material, acting as the waveguide boundaries. The dark core regions can be ascribed to the confinement of illumination light, indicating preliminarily their capability for light field restriction. This confinement originates from the RI contrast between the damage tracks (decreased RI) and the core regions (relatively high RI). The cores of the waveguides are kept intact and do not show evident cracks or damage, suggesting that guiding cores are expected to preserve faithful optical properties to the Ti: Sapphire substrate. As one can see from the top view image, the straight buses split unambiguously into two identical branches at the junctions. However, the branching areas are turned to be pitch-dark under optical microscope inspection due to the deceleration and acceleration that experience the 3D stage in order to change the scanning direction.

Fig. 3 shows the light intensity (normalized) distributions measured from the output face of the smallest splitter (Sp1). As one can see, this waveguide exhibits good splitting capability at both 632.8-nm laser (Fig. 3(a) and (b)) and 1064-nm radiation (Fig. 3 (c) and (d)) along two orthogonal polarizations, without strong light leakage through the waveguide barrier or, in particular, via the splitting point into the substrate regions. At 632.8-nm wavelength, the splitter is found to be quasi-single mode at both polarizations. One can expect a single-mode guidance at this wavelength by decreasing the RI contrast through, for example, further optimizing the writing parameters. In contrast, at 1064 $\mathrm{nm}$, mono-mode beam splitting is achieved under either polarization, which is an intriguing feature of this device for its application in photonic circuits. The circular or elliptical beam profiles of Sp1 at $1064 \mathrm{~nm}$ can be attribute to the single-mode behavior of the output arms. By carefully aligning the coupling arrangements, the output power through two arms of Sp1 at $1064 \mathrm{~nm}$ are measured and the ratio of 1-to- 2 beam splitting are determined to be around 1:0.92 and 1:1 under TE and TM polarizations, respectively, which in general indicates a equalization of the incident beams.

In order to determine the RI variation in laser-induced tracks, we reconstruct a three-dimensional spatial RI distribution with BeamPROP assuming a step-index configuration. By adjusting the RI value in a step of $0.1 \times 10^{-3}$, the disparity between the simulated and measured profile is gradually narrowed down. Ultimately, the RI contrast of the formed structure is determined to be $6.0 \times 10^{-3}$, with which the maximum agreement between the simulation and the experiment is obtained. It is worth to point out that this value of RI contrast applies to all structures fabricated in this work owing to the identical irradiation parameters. The beam profile evolution of 1064-nm light propagating (i.e., mode profiles at different positions) along Sp1 under TM polarization is also simulated based on the reconstructed spatial RI distribution. The simulated results are shown in Fig. 3(e), in which a clear beam-profile splitting can be observed.

In Fig. 4(a)-(f), the intensity distributions of splitters Sp2-Sp4 at $1064 \mathrm{~nm}$ along both TE and TM polarizations are illustrated respectively, which show good performance for beam splitting function even with the splitting angle as large as $2^{\circ}$. However, the behavior 


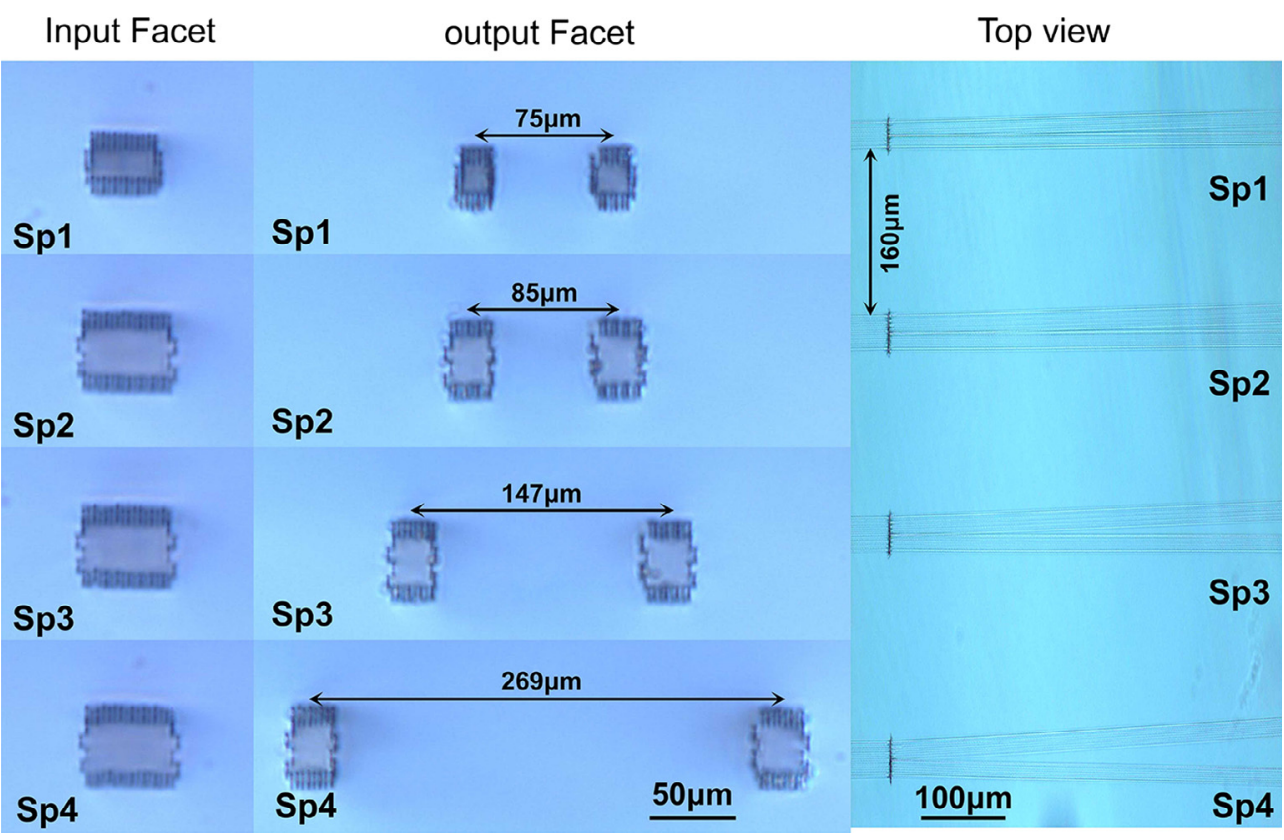

Fig. 2. Visible light transmission images of input facets, output facets and top view of cladding splitters Sp1-Sp4.
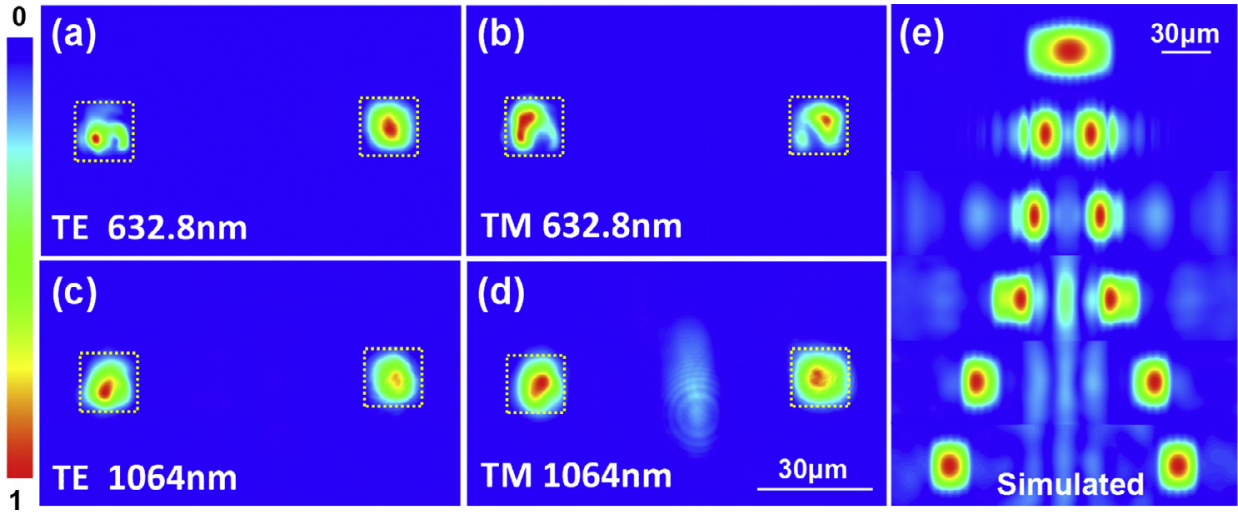

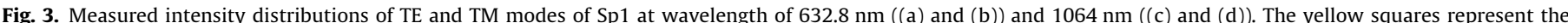

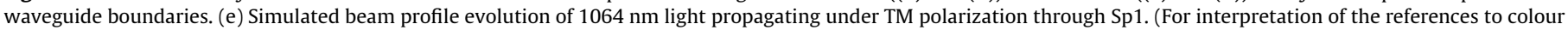
in this figure legend, the reader is referred to the web version of this article.)

of the waveguides is multimode at this wavelength, which can be attributed to the large size of the waveguide cores compared to $\mathrm{Sp} 1$. It can be found that, as the mode number increase, the modes would extend fully across the waveguide cross-section.

To further investigate the aforementioned polarization effect on cladding splitters, the all-angle powers through Sp1-Sp4 are measured at $1064 \mathrm{~nm}$. As shown in Fig. 5(a), the elliptical fittings of the resultant data indicate slight polarization sensitivity for all splitters. At $90^{\circ} / 270^{\circ}$, which corresponds to TM polarization, the maximum output powers are observed, while the minimum are obtained at $0^{\circ} / 180^{\circ}$ which correspond to TE polarization. These phenomena, in a first-order approximation, can be ascribed to the anisotropy of Ti:Sapphire crystal accompanied with the asymmetry RI profiles induced by FLI. Double-line waveguides in Ti:Sapphire has been previously reported, in which the guiding and lasing effects with a certain polarization are found [30]. Therefore, the splitters produced in this work are superior in term of all-angle 2D guidance. Based on the ratios of output power to the input power shown in Fig. 5(a), the total attenuations of these cladding splitters are determined, the results of which are plotted in Fig. 5 (b). For single-mode splitter (Sp1), the total losses are approximately $6.1 \mathrm{~dB}$ and $4.8 \mathrm{~dB}$ at TE and TM polarization. As the waveguiding volume is expanded, the losses of Sp2 reduce to $4.5 \mathrm{~dB}$ and $3.3 \mathrm{~dB}$ under TE and TM polarization, respectively. Additionally, with the enlargement of splitting angle, the splitting losses are increasing, leading to attenuations of $6.8 \mathrm{~dB}$ and $5.7 \mathrm{~dB}$ for $\mathrm{Sp} 4$ along TE and TM polarization. By adjusting the inscription parameters or optimizing waveguide structures (such as through thermal annealing treatment), the losses are expected to be further reduced.

Fig. 6(a) and (b) illustrate the 2D mappings of spatial dependence of the $\mu$-PL intensity emitted from the cross-sections of input and output facets of $\mathrm{Sp} 1$ correlated to $\mathrm{Ti}^{3+}$ ions at ${ }^{2} \mathrm{E} \rightarrow{ }^{2} \mathrm{~T}_{2}$ transition. From a first inspection it is clear that, the tracks are accompanied by a strong quenching in the fluorescence intensity. It has been found that the reduction in the luminescence intensity is related to a high density of lattice defects and imperfections occurred in the laser-induced tracks. These lattice defects and imperfections are also responsible to the negative RI change in the track [31]. In contrast, the waveguiding cores are composed of intact crystalline 

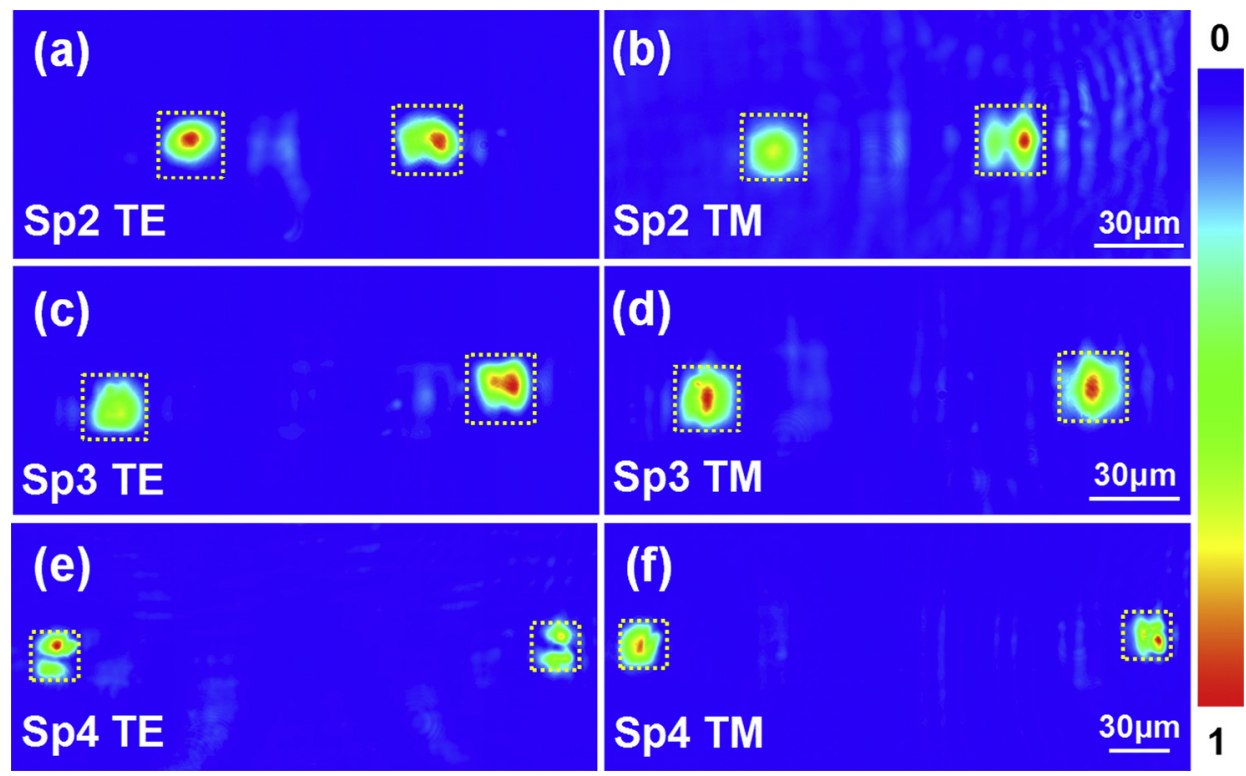

Fig. 4. (a)-(f) Intensity distribution of TE and TM modes of Sp2-Sp4 at $1064 \mathrm{~nm}$.
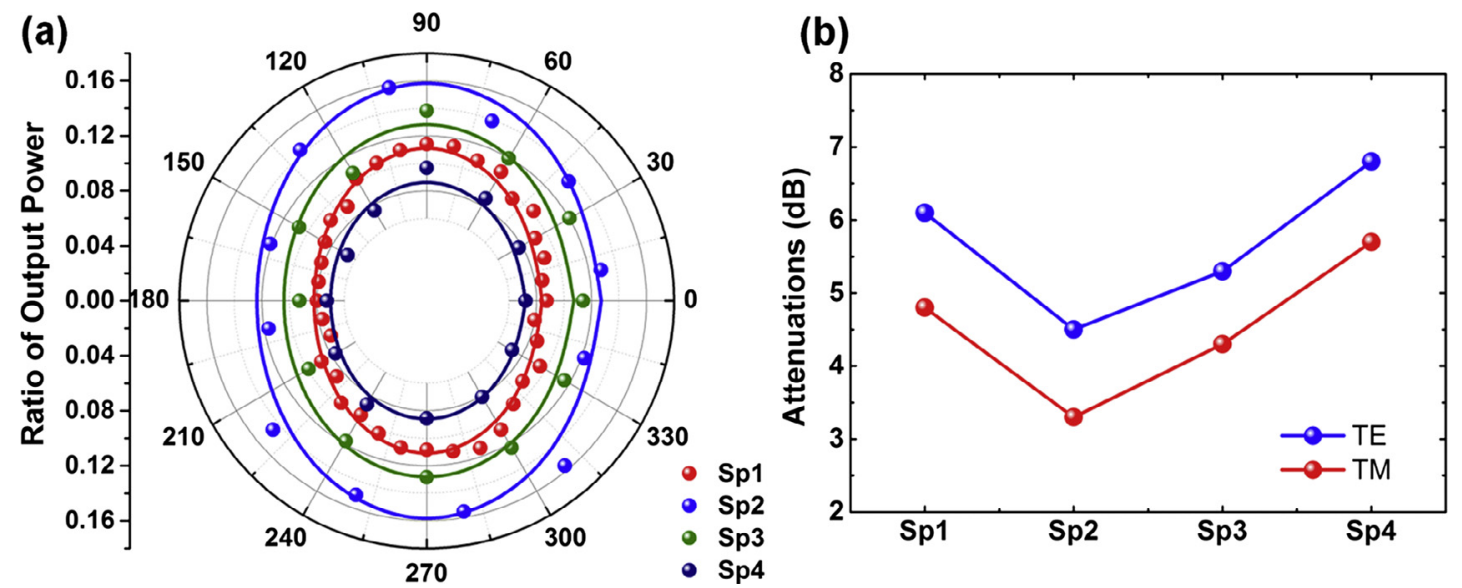

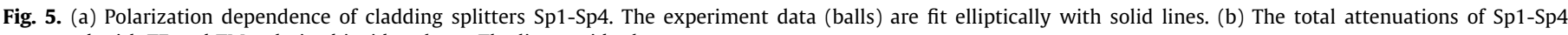
measured with TE and TM polarized incident laser. The lines guide the eye.

materials where the fluorescence properties of the sample is well preserved. Additionally, Fig. 6(c) depict the 1D distribution of the $\mu$-PL intensity measured along the dashed line (see insert of Fig. 6 (c)) crossing the input cross-section of Sp1. It is evidenced that the track centers have obvious fluorescence quenching (up to $20 \%$ intensity decrease with respect to the bulk). However, although slightly influenced by laser-induced tracks, the average fluorescence intensity obtained from the active volume is retained more than $95 \%$ of that generated from the original Ti:Sapphire network. Similar results can be obtained from Fig. 6(d) which includes the $\mu$-PL spectra ranging from $600 \mathrm{~nm}$ to $1000 \mathrm{~nm}$ collected from the bulk region, the track and the waveguide area. As can be seen, the spectra emitted from waveguide area is close to that obtained from the original bulk in terms of spectral profile and intensity, meanwhile, an obvious reduction of $\mu$-PL intensity in the laser-induced track is occurred. As a consequence, the guiding areas of the splitters produced in our work retain the good luminescence properties of the Ti:Sapphire crystal, revealing that the fabricated waveguide splitters are promising as integrated laser element.
Table 1 compares the performances of some 1-to-N splitters which are based on type I, type II and type III waveguides. These waveguides are fabricated by using FLI in transparent materials including glass, polymers and crystals. It can be seen that, the splitters produced in our work are of comparable performance. In addition, unlike the previously demonstrated superficial splitters in Nd: YAG [17] and $\mathrm{LiTaO}_{3}$ [29] crystals, in which a square input is split into two rectangular outputs, the output facets of the splitters in this work are square. This ensures the symmetry of the output modal profiles, therefore a high coupling efficiency with other optical devices or circuits can be achieved, making them advantageous in integrated photonics. Moreover, these built-in splitters are superior to the previously reported ones $[17,29]$ for building complex lab-on-chip photonic devices since more superficial networks can be introduced in the undamaged substrate surface. In combination with the unique optical properties of Ti:Sapphire crystal, the splitters reported in the work are promising for active applications as miniature photonic devices for the generation of broadly tunable laser. 

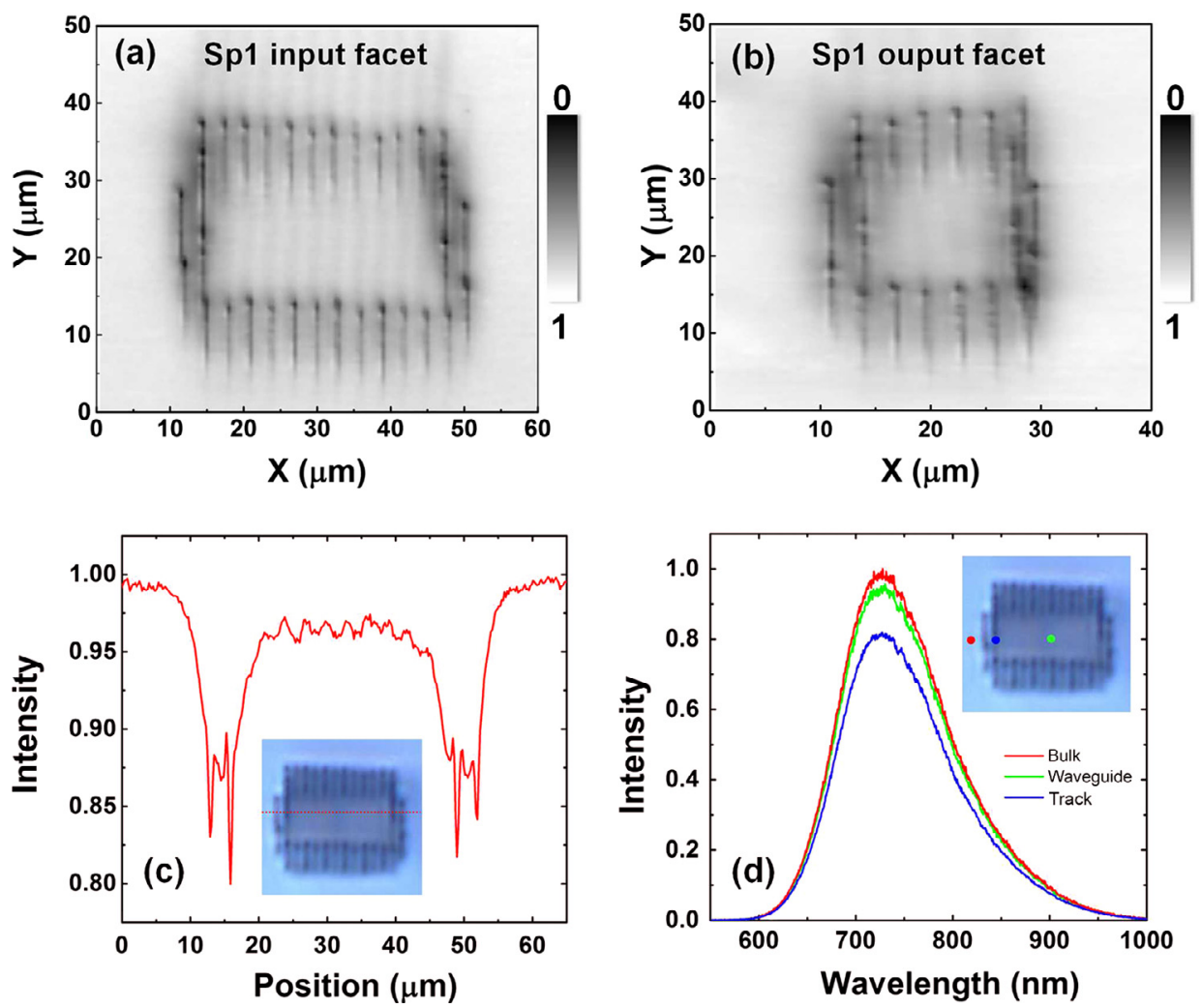

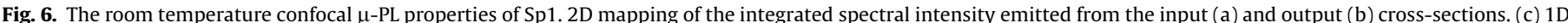

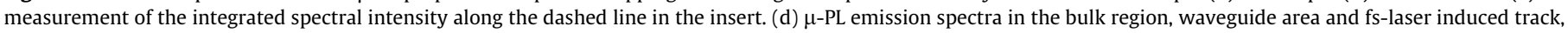

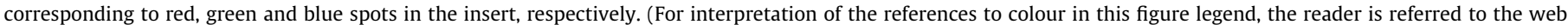
version of this article.)

Table 1

Comparison of some 1-to-N splitters based on type I, type II and type III wavegudes fabricated in different materials by using FLI.

\begin{tabular}{|c|c|c|c|c|c|}
\hline Material & FLI parameters ${ }^{a}$ & $\begin{array}{l}\text { Splitter } \\
\text { morphology }\end{array}$ & $\begin{array}{l}\text { Splitting angle/ } \\
\text { output separation }\end{array}$ & Propagating mode & Losses $(\mathrm{dB})$ \\
\hline $\begin{array}{l}\text { Borosilicate } \\
\text { glass [24] }\end{array}$ & $\begin{array}{l}1047 \mathrm{~nm}, 350 \mathrm{fs}, 500 \mathrm{kHz}, 165 \mathrm{~nJ}, 0.4 \mathrm{~N} . \mathrm{A} . \text {, circular } \\
\text { polarization, } 8.0 \mathrm{~mm} / \mathrm{s} \text {, below the surface }\end{array}$ & $\begin{array}{l}\text { Type I } \\
\text { (multi-scan) } \\
1 \times 16\end{array}$ & $50 \mu \mathrm{m}$ & Single-mode at $1539 \mathrm{~nm}$ & $\begin{array}{l}2.0 \text { at } 1539 \\
\mathrm{~nm}\end{array}$ \\
\hline PMMA [25] & $\begin{array}{l}1031 \mathrm{~nm}, 420 \mathrm{fs}, 1 \mathrm{MHz}, 185 \mathrm{~nJ}, 0.6 \mathrm{~N} . A ., 4 \mathrm{~mm} / \mathrm{s} \text { and } \\
6 \mathrm{~mm} / \mathrm{s}, \sim 200 \mu \mathrm{m} \text { below the surface }\end{array}$ & $\begin{array}{l}\text { Type I } \\
\text { (single-scan) } \\
1 \times 2,1 \times 4\end{array}$ & $0.353^{\circ}$ & Single-mode at $632.8 \mathrm{~nm}$ & $\begin{array}{l}7.23 \text { and } 8.62 \\
\text { at } 632.8 \mathrm{~nm}\end{array}$ \\
\hline $\begin{array}{l}\mathrm{LiNbO}_{3} \\
\quad \text { crystal } \\
\quad[26]\end{array}$ & $\begin{array}{l}1031 \mathrm{~nm}, 5 \mathrm{kHz}, 420 \mathrm{fs}, 4.9 \mu \mathrm{J}, 0.6 \mathrm{~N} . \mathrm{A} ., 4 \mathrm{~mm} / \mathrm{s}, \sim 150 \\
\mu \mathrm{m} \text { below the surface }\end{array}$ & $\begin{array}{l}\text { Type I } \\
\text { (single-scan) } \\
1 \times 2,1 \times 4\end{array}$ & $0.229^{\circ}$ & $\begin{array}{l}\text { At } 632.8 \mathrm{~nm} \text { and } 1064 \mathrm{~nm} \text { : single-mode } \\
\text { under TM no guidance under TE }\end{array}$ & $\begin{array}{l}3.45 \text { and } 3.61 \\
\text { at } 632.8 \mathrm{~nm}\end{array}$ \\
\hline $\begin{array}{r}\mathrm{Bi}_{4} \mathrm{Ge}_{3} \mathrm{O}_{12} \\
\text { crystal } \\
{[27]}\end{array}$ & $\begin{array}{l}800 \mathrm{~nm}, 120 \mathrm{fs}, 1 \mathrm{kHz}, 0.14 \mu \mathrm{J}, 0.4 \text { N.A., } 500 \mu \mathrm{m} / \mathrm{s}, \sim 50 \\
\mu \mathrm{m} \text { below the surface }\end{array}$ & $\begin{array}{l}\text { Type I } \\
\text { (multi-scan) } \\
1 \times 2,1 \times 3 \text {, } \\
1 \times 4\end{array}$ & $0.4^{\circ}-0.9^{\circ}$ & $\begin{array}{l}\text { Single-mode at } 4 \mu \mathrm{m} \text { polarization- } \\
\text { insensitive }\end{array}$ & $\begin{array}{l}3.23-3.95 \text { at } \\
4 \mu \mathrm{m}\end{array}$ \\
\hline $\begin{array}{c}\text { Yb:YAG } \\
\text { crystal } \\
{[28]}\end{array}$ & $\begin{array}{l}775 \mathrm{~nm}, 150 \mathrm{fs}, 0.65 \text { N.A, } 25 \mu \mathrm{m} / \mathrm{s}, \sim 300 \mu \mathrm{m} \text { below the } \\
\text { surface }\end{array}$ & $\begin{array}{l}\text { Type II } \\
1 \times 2\end{array}$ & $81-647 \mu \mathrm{m}$ & Multi-mode at $1030 \mathrm{~nm}$ & $\begin{array}{l}1.3-3 \text { at } 633 \\
\mathrm{~nm}\end{array}$ \\
\hline $\begin{array}{c}\text { Nd:YAG } \\
\text { crystal } \\
{[17]}\end{array}$ & $\begin{array}{l}795 \mathrm{~nm}, 120 \mathrm{fs}, 1 \mathrm{kHz}, 15 \mu \mathrm{J}, 0.6 \text { N.A., linear } \\
\text { polarization, } 500 \mu \mathrm{m} / \mathrm{s} \text {, on the surface }\end{array}$ & $\begin{array}{l}\text { Type III } \\
\text { (rectangular) } \\
1 \times 2\end{array}$ & $0.5^{\circ}-2^{\circ}$ & $\begin{array}{l}\text { Multi-mode at } 1064 \mathrm{~nm} \text { polarization- } \\
\text { insensitive }\end{array}$ & $\begin{array}{l}1.1 \text { at } 632.8 \\
\mathrm{~nm}\end{array}$ \\
\hline $\begin{array}{c}\mathrm{MgO}: \mathrm{LiTaO}_{3} \\
\text { crystal } \\
{[29]}\end{array}$ & $\begin{array}{l}795 \mathrm{~nm}, 120 \mathrm{fs}, 1 \mathrm{kHz}, 0.4 \mu \mathrm{J}, 0.4 \text { N.A., linear } \\
\text { polarization, } 500 \mu \mathrm{m} / \mathrm{s} \text {, on the surface }\end{array}$ & $\begin{array}{l}\text { Type III } \\
\text { (rectangular) } \\
1 \times 2\end{array}$ & $0.8^{\circ}-2.6^{\circ}$ & $\begin{array}{l}\text { Single-mode at } 1550 \mathrm{~nm} \text { polarization- } \\
\text { insensitive }\end{array}$ & \\
\hline $\begin{array}{c}\text { Ti:Sapphire } \\
\text { (this } \\
\text { work) }\end{array}$ & $\begin{array}{l}795 \mathrm{~nm}, 120 \mathrm{fs}, 1 \mathrm{kHz}, 1.2 \mu \mathrm{J}, 0.4 \mathrm{~N} . A ., \text { linear } \\
\text { polarization, } 500 \mu \mathrm{m} / \mathrm{s}, \sim 260 \mu \mathrm{m} \text { below the surface }\end{array}$ & $\begin{array}{l}\text { Type III } \\
\text { (rectangular) } \\
1 \times 2\end{array}$ & $0.5^{\circ}-2^{\circ}$ & $\begin{array}{l}\text { Single-mode at1064nm (Sp1) } \\
\text { polarization-insensitive }\end{array}$ & $\begin{array}{l}3.3-6.8 \text { at } \\
1064 \mathrm{~nm}\end{array}$ \\
\hline
\end{tabular}

${ }^{a}$ FLI parameters are listed in order of fs-laser wavelength, pulse duration, repetition rate, pulse energy, focusing N.A., laser polarization (if provided), scan speed, waveguide location. 


\section{Conclusion}

In conclusion, waveguide splitters with different dimensions and divergence angles are produced inside a Ti:Sapphire crystal by FLI, based on depressed-cladding structures. Each splitter is designed to have a rectangular input arm that splits equally into two square exiting arms, giving symmetric output profiles. These splitters exhibit strong light confinement and equalization of the output power for 1-to-2 beam splitting. In particular, singlemode guidance is obtained from the smallest splitter. Furthermore, the splitters show a low dependence of the guiding properties with the polarization of the incident laser beam. The confocal $\mu$-PL measurements reveal that the luminescence properties of Ti:Sapphire network in the waveguide volumes are well preserved. The good performances of these waveguiding splitters make them promising candidates in lab-on-a-chip photonic circuits and miniature optical devices.

\section{Acknowledgements}

This work is supported by the National Natural Science Foundation of China (No. 11404194). Authors acknowledge support from Junta de Castilla y León (Project SA046U16) and MINECO (FIS2015-71933-REDT). Authors would like to thank Prof. Xiaotao Hao from Shandong University for the help on microphotoluminescence measurement.

\section{References}

[1] G. Lifante, Integrated Photonics: Fundamentals, John Wiley \& Sons, Chichester, 2008

[2] F. Chen, Micro- and submicrometric waveguiding structures in optical crystals produced by ion beams for photonic applications, Laser Photon. Rev. 6 (2012) 622-640.

[3] R. Keil, M. Heinrich, F. Dreisow, T. Pertsch, A. Tünnermann, S. Nolte, D.N. Christodoulides, A. Szameit, All-optical routing and switching for threedimensional photonic circuitry, Sci. Rep. 1 (2011) 94.

[4] G.D. Marshall, A. Politi, J.C.F. Matthews, P. Dekker, M. Ams, M.J. Withford, J.L. O'Brien, Laser written waveguide photonic quantum circuits, Opt. Express 17 (2009) 12546-12554.

[5] Y. Yang, A.Q. Liu, L.K. Chin, X.M. Zhang, D.P. Tsai, C.L. Lin, C. Lu, G.P. Wang, N.I. Zheludev, Optofluidic waveguide as a transformation optics device for lightwave bending and manipulation, Nat. Commun. 3 (2012) 651.

[6] R.R. Thomson, R.J. Harris, T.A. Birks, G. Brown, J. Allington-Smith, J. BlandHawthorn, Ultrafast laser inscription of a 121-waveguide fan-out for astrophotonics, Opt. Lett. 37 (2012) 2331-2333.

[7] Y.C. Jia, C. Cheng, J.R. Vázquez de Aldana, G.R. Castillo, B. del Rosal Rabes, Y. Tan, D. Jaque, F. Chen, Monolithic crystalline cladding microstructures for efficient light guiding and beam manipulation in passive and active regimes, Sci. Rep. 4 (2014) 5988.

[8] C. Grivas, Optically pumped planar waveguide lasers: part II: gain media, laser systems, and applications, Prog. Quant. Electron. 45-46 (2016) 3-160.

[9] D.P. Shepherd, A. Choudhary, A.A. Lagatsky, P. Kannan, S.J. Beecher, R.W. Eason, J.I. Mackenzie, X. Feng, W. Sibbett, C. Tom, A. Brown, Ultrafast high-repetitionrate waveguide lasers, IEEE J. Sel. Topic Quant. Electron. 22 (2015) 1100109.

[10] Y. Tan, O.F. Luan, F.O. Liu, S. Akhmadaliev, S.O. Zhou, F. Chen, Swift carbon ion irradiated Nd:YAG ceramic optical waveguide amplifier, Opt. Express 21 (2013) 13992-13997.
[11] B. Albrecht, P. Farrera, X. Fernandez-Gonzalvo, M. Cristiani, H. de Riedmatten, A waveguide frequency converter connecting rubidium-based quantum memories to the telecom C-band, Nat. Commun. 5 (2014) 3376.

[12] F. Chen, J.R. Vázquez de Aldana, Optical waveguides in crystalline dielectric materials produced by femtosecond laser micromachining, Laser Photon. Rev. 8 (2014) 251-275

[13] J. Gamet, G. Pandraud, Field-matching Y-branch for low loss power splitter Opt. Commun. 248 (2005) 423-429.

[14] H. Jin, F.M. Liu, P. Xu, J.L. Xia, M.L. Zhong, Y. Yuan, J.W. Zhou, Y.X. Gong, W Wang, S.N. Zhu, On-chip generation and manipulation of entangled photons based on reconfigurable lithium-niobate waveguide circuits, Phys. Rev. Lett. 113 (2014) 103601.

[15] K.B. Mogensen, Y.C. Kwok, J.C.T. Eijkel, N.J. Petersen, A. Manz, J.P. Kutter, A microfluidic device with an integrated waveguide beam splitter for velocity measurements of flowing particles by fourier transformation, Anal. Chem. 75 (2003) 4931-4936.

[16] J.B. Driscoll, R.R. Grote, B. Souhan, J.I. Dadap, M. Lu, R.M. Osgood, Asymmetric Y junctions in silicon waveguides for on-chip mode-division multiplexing, Opt. Lett. 38 (2013) 1854-1856.

[17] H.L. Liu, J.R. Vázquez de Aldana, M.H. Hong, F. Chen, Femtosecond laser inscribed Y-branch waveguide in Nd:YAG crystal: fabrication and continuouswave lasing, IEEE J. Sel. Topic Quant. Electron. 22 (2016) 4500204.

[18] D. Du, X. Liu, G. Korn, J. Squier, G. Mourou, Laser-induced breakdown by impact ionization in $\mathrm{SiO}_{2}$ with pulse widths from 7 ns to $150 \mathrm{fs}$, Appl. Phys. Lett. 64 (1994) 3071-3073.

[19] R.R. Gattass, E. Mazur, Femtosecond laser micromachining in transparent materials, Nat. Photon. 2 (2008) 219-225.

[20] R. Osellame, H.J.W.M. Hoekstra, G. Cerullo, M. Pollnau, Femtosecond laser microstructuring: an enabling tool for optofluidic lab-on-chips, Laser Photon. Rev. 5 (2011) 442-463.

[21] K. Sugioka, Y. Cheng, Ultrafast lasers-reliable tools for advanced materials processing, Light Sci. Appl. 3 (2014) e149.

[22] D. Choudhury, J.R. Macdonald, A.K. Kar, Ultrafast laser inscription: perspectives on future integrated applications, Laser Photon. Rev. 8 (2014) 827-846.

[23] S. Eaton, C. Marco, R. Martinez-Vazquez, R. Ramponi, S. Turri, G. Cerullo, R. Osellame, Femtosecond laser microstructuring for polymeric lab-on-chips, J. Biophoton. 5 (2012) 687-702.

[24] R.R. Thomson, T.A. Birks, S.G. Leon-Saval, A.K. Kar, J. Bland-Hawthorn, Ultrafast laser inscription of an integrated photonic lantern, Opt. Express 19 (2011) 5698-5705.

[25] W.H. Yuan, J.M. Lv, C. Cheng, X.T. Hao, F. Chen, Waveguides and proportional beam splitters in bulk poly(methyl methacrylate) produced by direct femtosecond-laser inscription, Opt. Mater. 49 (2015) 110-115.

[26] J.M. Lv, Y.Z. Cheng, W.H. Yuan, X.T. Hao, F. Chen, Three-dimensional femtosecond laser fabrication of waveguide beam splitters in $\mathrm{LiNbO}_{3}$ crystal, Opt. Mater. Express 5 (2015) 1274-1280.

[27] R.Y. He, I. Hernández-Palmero, C. Romero, J.R. Vázquez de Aldana, F. Chen, Three-dimensional dielectric crystalline waveguide beam splitters in midinfrared band by direct femtosecond laser writing, Opt. Express 22 (2014) 31293-31298.

[28] T. Calmano, C. Kränkel, Günter Huber, Laser oscillation in Yb:YAG waveguide beam-splitters with variable splitting ratio, Opt. Lett. 40 (2015) 1753-1756.

[29] C. Cheng, C. Romero, J.R. Vázquez de Aldana, F. Chen, Superficial waveguide splitters fabricated by femtosecond laser writing of $\mathrm{LiTaO}_{3}$ crystal, Opt. Eng. 54 (2015) 067113.

[30] C. Grivas, C. Corbari, G. Brambilla, P.G. Lagoudakis, Tunable, continuous-wave Ti:sapphire channel waveguide lasers written by femtosecond and picosecond laser pulses, Opt. Lett. 37 (2012) 4630-4632.

[31] A. Ródenas, G.A. Torchia, G. Lifante, E. Cantelar, J. Lamela, F. Jaque, L. Roso, D. Jaque, Refractive index change mechanisms in femtosecond laser written ceramic Nd:YAG waveguides: micro-spectroscopy experiments and beam propagation calculations, Appl. Phys. B 95 (2009) 85-96. 